\title{
Neurofilament Phosphorylation during Development and Disease: Which Came First, the Phosphorylation or the Accumulation?
}

\author{
Jeffrey M. Dale $e^{1,2}$ and Michael L. Garcia ${ }^{1,2}$ \\ ${ }^{1}$ Division of Biological Sciences, University of Missouri-Columbia, Columbia, MO 65211, USA \\ ${ }^{2}$ Bond Life Sciences Center, University of Missouri-Columbia, Columbia, MO 65211, USA \\ Correspondence should be addressed to Michael L. Garcia, garciaml@missouri.edu
}

Received 17 November 2011; Accepted 31 January 2012

Academic Editor: Ewa A. Bienkiewicz

Copyright ( 2012 J. M. Dale and M. L. Garcia. This is an open access article distributed under the Creative Commons Attribution License, which permits unrestricted use, distribution, and reproduction in any medium, provided the original work is properly cited.

\begin{abstract}
Posttranslational modification of proteins is a ubiquitous cellular mechanism for regulating protein function. Some of the most heavily modified neuronal proteins are cytoskeletal proteins of long myelinated axons referred to as neurofilaments (NFs). NFs are type IV intermediate filaments (IFs) that can be composed of four subunits, neurofilament heavy (NF-H), neurofilament medium (NF-M), neurofilament light (NF-L), and $\alpha$-internexin. Within wild type axons, NFs are responsible for mediating radial growth, a process that determines axonal diameter. NFs are phosphorylated on highly conserved lysine-serine-proline (KSP) repeats located along the C-termini of both NF-M and NF-H within myelinated axonal regions. Phosphorylation is thought to regulate aspects of NF transport and function. However, a key pathological hallmark of several neurodegenerative diseases is ectopic accumulation and phosphorylation of NFs. The goal of this review is to provide an overview of the posttranslational modifications that occur in both normal and diseased axons. We review evidence that challenges the role of KSP phosphorylation as essential for radial growth and suggests an alternative role for NF phosphorylation in myelinated axons. Furthermore, we demonstrate that regulation of NF phosphorylation dynamics may be essential to avoiding NF accumulations.
\end{abstract}

\section{Introduction}

The established role of neurofilaments (NFs) is to increase axonal diameter in myelinated fibers thereby increasing nerve conduction velocity [1]. NFs are composed of an N-terminal head, central rod, and C-terminal tail domain [2] (Figure 1). NFs are posttranslationally modified in all three of these functional domains [3-7]. NF phosphorylation, the most frequent posttranslational modification (PTM) and focus of our review, occurs primarily at conserved KSXXP motifs (KSP) located on the C-terminal tail domain of neurofilament heavy (NF-H) and medium (NF-M) [3-6]. However, phosphorylation of "non-KSP" serine residues within NF$\mathrm{M}$ and neurofilament light (NF-L) amino terminal (Nterminal) head domain has been observed [7]. Transgenic [8] and gene-targeted [9] mouse lines expressing mutagenized NF phosphorylation sites have significantly advanced our understanding of the role of NF phosphorylation.
For example, mice expressing a serine to aspartate mutation at position 55 of NF-L, NF-L ${ }^{555}$, displayed accumulations of phosphorylated NFs within cell bodies [8]. Furthermore, mice expressing serine to alanine mutations within the 7 identified KSP motifs of NF-M, NF-M ${ }^{S \rightarrow A}$, demonstrated an unaltered distribution of axonal diameters [9].

Over the last 20 years, great strides have been taken in the characterization of NF proteins, their function, and their PTMs. In this review, we will attempt to describe the experiments that have led to the current understanding of the role of NF proteins and their PTMs in normal and diseased axons. Furthermore, using other intermediate filaments as a reference, we will detail a potential role for NF phosphorylation. Moreover, we will address the importance of investigating this putative role of NF phosphorylation and its potential to explain abnormal NF accumulations in neurodegenerative diseases. 


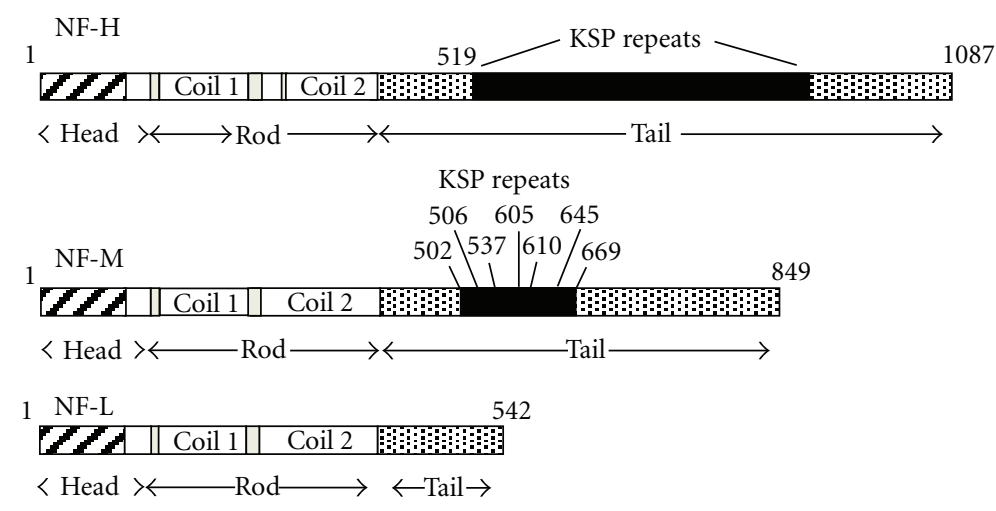

FIGURE 1: Representation of the functional domains of each NF subunit. The subdomains within each domain are identified along with approximate location of all relevant amino acid positions.

\section{Does KSP Phosphorylation Regulate Axon Diameter?}

Axonal diameter influences the rate of neuronal conduction velocity with larger caliber axons conducting faster than small caliber axons [10-12]. In myelinated axons, the internode (myelinated) has a larger diameter than nodes of Ranvier (unmyelinated). Axonal diameter is established by a process referred to as radial axonal growth. Axonal NFs were required for radial growth $[13,14]$. NF phosphorylation and radial growth were reduced in axons when compact myelin failed to form [15]. Moreover, phosphorylation of NFs Ctermini was increased in myelinated axonal regions relative to unmyelinated regions of the same axon [16, 17]. Taken together, these data suggested that KSP repeat phosphorylation was required for establishing axonal diameter.

Deletion of NF-M $[18,19]$ resulted in a reduction in radial growth that was similar to nerves without axonal NFs [14], whereas deletion of NF-H [20-22] did not reduce axonal diameter to a similar level as loss of NF-L [14] or NF-M $[18,19]$. Moreover, axonal diameter was reduced in mice expressing C-terminally truncated NF-M (NF-M Tail $)$ or C-terminally truncated NF-M and NF-H $\left(\mathrm{NF}-(\mathrm{M} / \mathrm{H})^{\mathrm{Tail}} \mathrm{s}\right)$ [23] (Figures 2(a) and 2(b)). However, truncation of NF$\mathrm{H}\left(\mathrm{NF}-\mathrm{H}^{\mathrm{Tai}} \Delta\right)$ only reduced axonal diameter in young mice [22]. Axonal diameters in older NF- $\mathrm{H}^{\text {Tail } \Delta}$ mice were similar to wild type [22]. These data suggested that NF-M and its C-terminus were essential for radial growth and seemed to support the NF KSP phosphorylation hypothesis of radial growth.

A direct test of this hypothesis was performed by expressing a KSP phosphoincompetent variant of NF-M in mice. Site-directed mutagenesis was utilized to mutate all KSP serine residues to alanine preventing KSP phosphorylation $\left(\mathrm{NF}-\mathrm{M}^{\mathrm{S}} \rightarrow \mathrm{A}\right.$ mice) without removing the entire C-terminus of NF-M [9] (Figure 2(c)). Surprisingly, axonal diameter was not altered in NF-M ${ }^{S \rightarrow A}$ mice suggesting that KSP phosphorylation was not an essential component of radial axonal growth [9] (Figure 2(c)). When taken together with the results obtained from NF-M $\mathrm{M}^{\mathrm{Tai} \Delta}$ mice, these results suggested that the C-terminus of NF-M mediated radial growth by a mechanism that did not require KSP phosphorylation. Therefore, the precise mechanism by which NF-M C-terminus mediates radial growth has yet to be determined.

\section{Proximal to Distal Appearance of Phospho-Epitopes of NFs during Axonal Transport}

Although NF phosphorylation does not directly regulate radial growth, evidence suggested a progressive appearance of phosphoepitopes $[7,24,25]$ as NFs are transported from the neuronal cell bodies to the axons [26]. Radiolabeling of NFs in retinal ganglion cells of mice suggested that NFs were more heavily phosphorylated in distal axonal regions [25]. Moreover, the appearance of phosphoepitopes on NF proteins occurred at distinct developmental stages. NF-H phosphorylation resulted in the appearance of a phosphoepitope that was recognized by the SMI-34 monoclonal antibody [27]. As NFs enter the optic nerve, this epitope was readily visualized suggesting amino acid phosphorylation that contributes to the SMI-34 epitope occurred in neuronal cell bodies [28]. A phosphoepitope recognized by SMI-31 on NF-H was detected only within axons of the optic nerve subsequent to the SMI-34 epitope [28], and the phosphoepitope recognized by RT97 antibodies was detected last and was only evident in axons of optic nerve [28]. Appearance of the RT97 epitope coincided with local accumulation of NFs and initiation of radial growth [28]. Taken together these results suggested that temporally distinct phosphorylation events of NF proteins regulated NF transport in optic nerve axons.

\section{Multiple Causes of Aberrant NF Accumulations}

NF accumulations are hallmarks of neurodegenerative diseases (NDDs). However, it is unclear how NDDs alter NF dynamics resulting in the observed accumulations. Analysis of NFs suggests several potential sites where disease-induced alterations could result in NF accumulations. One such 


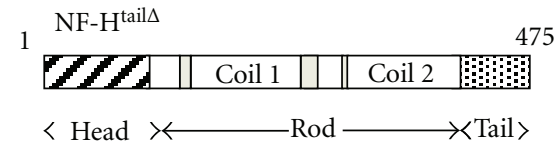

(a)

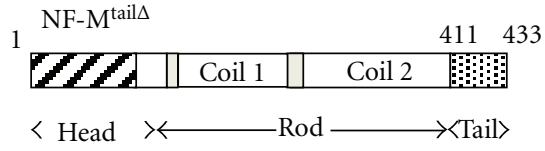

(b)

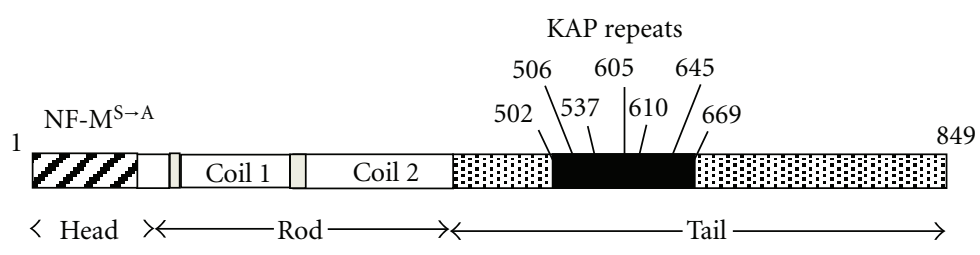

(c)

Figure 2: Representation of NF-H and NF-M mutants. (a) Incorporation of a C-terminal myc tag results in loss of 612 amino acids including all KSP repeat motifs. (b) Incorporation of a C-terminal myc tag results in loss of 426 amino acids including 7 KSP repeat motifs. (c) Sitedirected mutagenesis was utilized to mutate all KSP serine residues to alanine thereby preventing KSP serine phosphorylation without deletion of the remaining amino acids. The approximate location of each KSP repeat is identified.

mechanism is disrupted NF transport and altered local accumulation caused by altered phosphorylation dynamics.

Mechanistically, phosphorylation may regulate NF transport by altering NF association with molecular motors. Phosphorylation of NF-H and NF-M altered NF association with kinesin [29, 30] and dynein [26]. Decreased NF-H association with the anterograde motor, kinesin, coincided with the appearance of the C-terminal RT97 phopho-epitope [31] and correlated with increased NF-H association with the retrograde motor, dynein [26]. Taken together, these data suggest that increased C-terminal (KSP) phosphorylation directed the overall flow of NF transport towards neuronal cell bodies providing evidence for a role of altered phosphorylation dynamics in the appearance of phosphorylated NF accumulations within motor neuron cell bodies.

Moreover, deletion of either NF-H [19-21] or NF-M [32] increased the rate of NF transport in vivo supporting a role for KSP phosphorylation in regulating NF transport. Therefore, increased rates of NF transport may have resulted from increased association of NFs with kinesin, if loss of a single subunit results in a net loss of phosphorylation. Interestingly, deletion of NF C-termini and all KSP repeats did not alter NF transport rates $[22,33,34]$. Unaltered rates of NF transport in mice expressing truncated NFs appeared to contradict a primary role for NF phosphorylation in regulating NF transport. However, truncation of a single subunit resulted in compensatory phosphorylation of the remaining subunit $[22,23]$. Thus, phosphodependent regulation of NF transport cannot be completely ruled out until transport is measured in mice simultaneously expressing C-terminally truncated NF-M and NF-H.

NF-L [35] and NF-M [7] were phosphorylated at serine residues located throughout their amino termini, especially serine ${ }^{55}$. Mimicking constitutive phosphorylation by mutating serine residues to aspartate, such as NF- $\mathrm{L}^{\mathrm{S} 55 \mathrm{D}}$, altered NF assembly and decreased NF transport [35]. Transgenic mice expressing low levels of NF-L ${ }^{\text {S5DD }}$ developed
NF accumulations within neuronal cell bodies in mice as young as 4 weeks old [8]. Additionally, phosphorylation of NF-M at protein kinase A sites within the N-terminus inhibited C-terminal KSP phosphorylation of NF-M [7]. Thus, phosphorylation of NF-L and NF-M on serine residues located within the $\mathrm{N}$-terminus may be a mechanism to delay NF assembly and phosphorylation thereby preventing ectopic accumulation of NFs within neuronal cell bodies.

Altering NF subunit stoichiometry by overexpression of NF-L [36, 37], NF-M [36], or NF-H [38] altered NF phosphorylation dynamics. NF-L and NF-H overexpression resulted in phosphorylated NF accumulations in motor and sensory neuron cell bodies that were not observed in control littermates $[37,38]$. NF-M overexpression resulted in decreased NF-H phosphorylation and increased NF-L expression [36]. Furthermore, NF-L or NF-H overexpression resulted in morphological abnormalities, cell body swelling, and muscle atrophy that were similar to the pathologies observed in amyotrophic lateral sclerosis (ALS) [39, 40]. Neuronal pathology was alleviated in NF-H transgenic mice by simultaneous overexpression of NF-L [41] highlighting the importance of regulating the relative stoichiometry of the individual NF subunit proteins. Altered subunit stoichiometry has been observed in NDDs. NF-L mRNA was selectively decreased in Alzheimer's disease (AD) [42]. NFL mRNA expression was also reduced in sporadic [43] and superoxide dismutase 1-linked familial ALS [44]. Deletion of NF-L in mice resulted in accumulation of NF-H and NF-M in neuronal cell bodies [45]. If reductions in NF-L mRNA expression lead to reduced NF-L protein, then, as observed in genetically altered mice, altering NF subunit stoichiometry may contribute to NF accumulations observed in both $\mathrm{AD}$ and ALS.

A potential mechanism for regulating NF content within the axon is degradation by the ubiquitin proteasome system (UPS) [46-48]. Disruption of two components of the UPS, tripartite motif protein 2 (Trim $2^{\mathrm{GT}}$ ) mice [46] and ubiquitinspecific protease 14 (Usp14) ( $\mathrm{ax}^{\mathrm{J}}$ mice) [49-51], resulted 
in phenotypic ataxia resulting from motor neuron dysfunction. Upon further examination, Trim $2^{\mathrm{GT}}$ mice displayed alterations associated with neurodegeneration [46]. Onemonth-old Trim $2^{\mathrm{GT}}$ mice contained axonal swellings and NF accumulations in regions that overlapped calbindin D-28K immunoreactivity suggesting that the axons originated from Purkinje neurons [46]. By 5 months, Purkinje cell loss was readily apparent. Trim $2{ }^{\mathrm{GT}}$ mice showed an $85 \%$ reduction in the number of Purkinje cells relative to control mice [46].

$\mathrm{Ax}^{\top}$ mice demonstrated a selective loss of large caliber axons within the fourth and fifth lumbar (L4-L5) motor roots [49]. Furthermore, $\mathrm{Ax}^{\mathrm{J}}$ mice displayed severe neuromuscular junction (NMJ) defects such as swollen and poorly arborized nerve terminals that demonstrated sprouting, denervation, and disorganized postsynaptic AChRs [49]. Within the tibialis anterior (TA) muscle, axonal swellings contained NF accumulations that were observed as early as P7 [49]. Moreover, NMJ defects were accompanied by defects in synaptic transmission. $\mathrm{Ax}^{\mathrm{J}}$ mice had a 1.5 -fold increase in miniature end-plate potential (mEPP) amplitude. Additionally, mEPP frequency and vesicular quantal content were both reduced by $\sim 40 \%$ suggesting synaptic vesicle disorganization $[49,50]$.

Disruptions to both components of the UPS resulted in axonal swellings containing phosphorylated NF accumulations. Trim 2 expression was almost exclusively limited to the nervous system in Trim $2^{\mathrm{GT}}$ mice [46]. Moreover, restoration of neuronal Usp14 levels (Thy1-Usp transgene) in ax ${ }^{J}$ mice alleviated phenotypic ataxia [52] and NF accumulations [49]. Therefore, altering the neuronal UPS resulted in NF accumulations that were similar to those observed in mice expressing NFs that mimic constitutive phosphorylation within the N-terminal head domain, mice with altered NF stoichiometry, and many human NDDs.

\section{Is NF Phosphorylation a Mechanism to Enhance Stability by Reducing NF Degradation?}

NFs are long-lived axonal proteins whose accumulation within the axon is strictly regulated. Although evidence suggests that NFs associate with molecular motors [26, 29, 30] in a phosphodependent manner to reach their destination, little is known about the mechanisms that prevent excessive NF accumulation and regulate local NF turnover. The appearance of NF accumulations in axons with UPS defects suggests that the UPS has a critical role in the regulation of NF turnover.

Ubiquitin epitopes have been identified on NF-M purified from spinal cord verifying that ubiquitination is indeed NF PTM [48]. Furthermore, NF-L has been identified as a target of the E3 enzyme, Trim2 [46], and NF-M has been identified as a target of the E3 enzyme, carboxyl-terminus of Hsc70 interacting protein (CHIP) [47]. Identification of NF E3 enzymes supports the claim that NFs may undergo ubiquitin-mediated degradation. However, the mechanisms responsible for regulating NF ubiquitination have yet to be determined. Trim2 and CHIP are classified as E3 ligases
$[46,47]$ which are proteins that catalyze the transfer of ubiquitin to substrate proteins [53]. Both Trim2 [54] and CHIP [55] targeted and ubiquitinated protein substrates in a phosphodependent manner. The E3 ligase, c-Cbl, had a similar phosphodependence for its interaction with epidermal growth factor receptor (EGFR) and sprouty-2 (Spry2) [56]. Interestingly, increased phosphorylation of EGFR or Spry2 reduced $\mathrm{c}-\mathrm{Cbl}$ substrate binding [56]. Although evidence suggested that Trim 2 and CHIP require substrate phosphorylation $[54,55]$, it is unknown if increased phosphorylation would negatively regulate TRIM2 and CHIP affinity for NF subunits similar to $\mathrm{c}$-Cbl. However, evidence suggested that NF stability is increased with increasing phosphorylation. NF phosphorylation was increased in cells [57] and brain slices [58] treated with the phosphatase inhibitor, okadaic acid, resulting in increased NF levels $[57,58]$. Furthermore, treating purified NFs with alkaline phosphatase resulted in dephosphorylated NFs that were degraded more rapidly by the calcium dependent protease, calpain, when compared to phosphorylated NFs [59]. Therefore, increased NF phosphorylation may be a mechanism for enhancing NF stability by preventing NF turnover by UPS or protease-dependent mechanisms.

Ubiquitination of the type I/II intermediate filaments, keratins, may offer insight into the interaction between NF phosphorylation and ubiquitination. Like NFs, keratins contain a consensus sequence $\left({ }^{69} \mathrm{QSLLSPL}^{75}\right)$ that is phosphorylated. A proline to leucine mutation at position 74 of keratin $8, \mathrm{~K}^{\mathrm{P} 74 \mathrm{~L}}$, eliminated the phosphorylation of this consensus site and increased K8 ubiquitination [60]. Similarly, $\mathrm{K} 8^{\mathrm{P} 74 \mathrm{~A} / \mathrm{D}}$ mutations led to decreased phosphorylation and increased $\mathrm{K} 8$ ubiquitination [60]. Conversely, a $\mathrm{K} 8{ }^{\mathrm{L} 71 \mathrm{P}}$ mutation increased $\mathrm{K} 8$ phosphorylation and decreased K8 ubiquitination [60]. Taken together these data suggest that phosphorylation and ubiquitination of $\mathrm{K} 8$ are reciprocally regulated $[61,62]$. The similarity between NFs (type IV intermediate filaments) and keratins (type I/II intermediate filaments) suggests that NF KSP phosphorylation could regulate subsequent PTMs in a manner similar to keratin phosphomotifs.

\section{NF Phosphorylation Is Increased in Neurodegenerative Diseases}

In many NDDs, accumulations of phosphorylated NFs are hallmarks of pathology. Increased phosphorylation resulted in increased NFs $[57,58]$ and decreased K8 ubiquitination [60], whereas dephosphorylation of the most heavily phosphorylated NF subunit, NF-H, increased its turnover sixfold [59]. Furthermore, NF-L [46] and NF-M [47] have recently been identified as E3 ligase targets. Taken together, these data suggest that ectopic phosphorylation of NFs results in NF accumulations, which may lead to further phosphorylation subsequent to aggregate formation. Delineating the mechanisms that regulate NF phosphorylation may prove to be critical in regulating NF accumulations in NDDs. In this section, we focus on three NDDs as models of NF contribution to disease pathogenesis. 
CMT2E-linked NF-L mutations

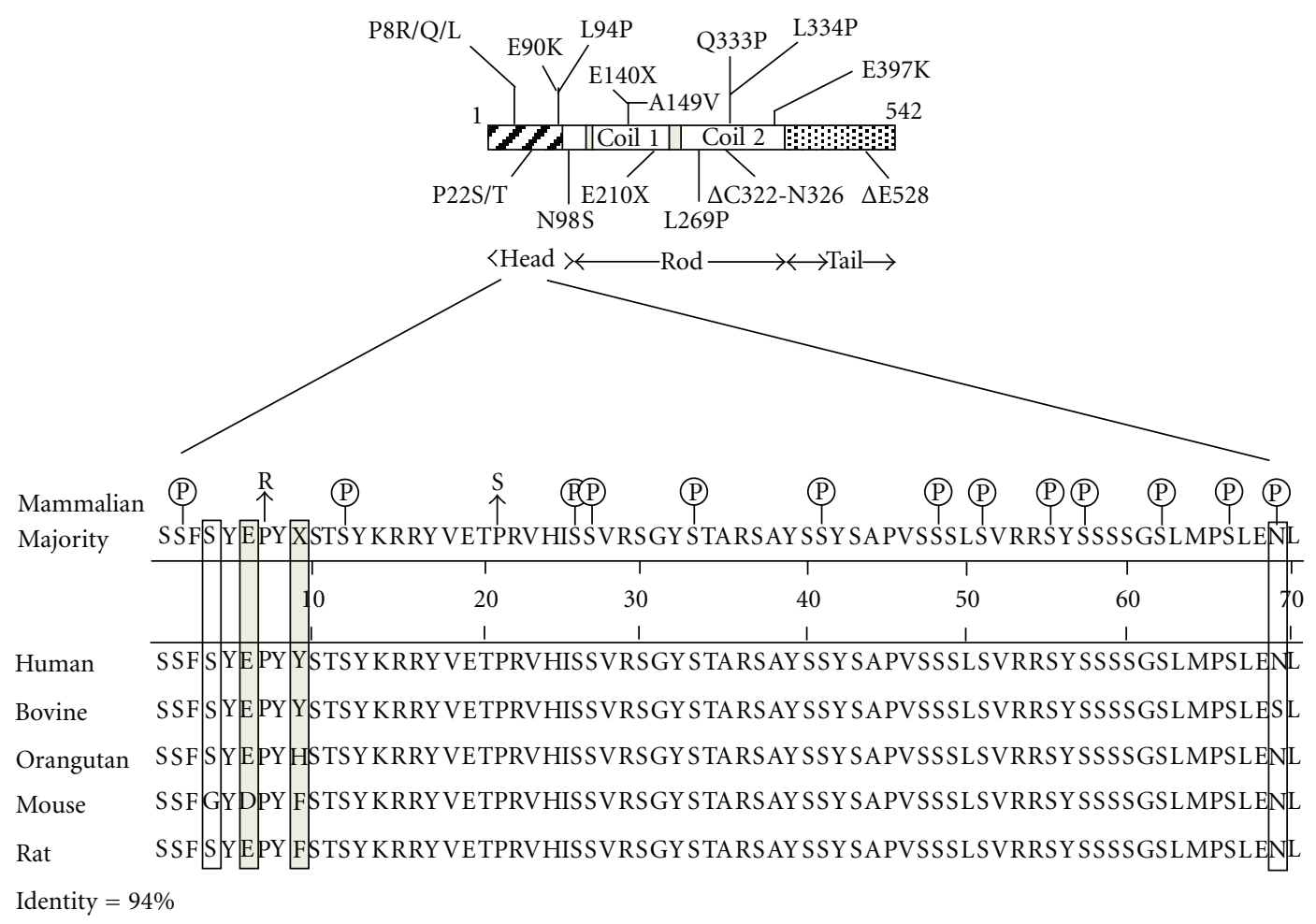

FIGURE 3: Relative positions of CMT2E-linked NF-L mutations and NF-L head domain phosphorylation sites. (A) Identity and location of all identified CMT2E-linked NF-L mutations. (B) Aligned protein sequences of human, bovine, orangutan, murine, and rat NF-L Nterminal head domains. Identified serine phosphorylation sites within bovine NF-L are indicated along the consensus sequence. Shaded boxes indicate conserved sequence variations, and empty boxes identify nonconserved sequence variations. An X in the consensus sequence identifies amino acid positions that lack an overall consensus between the five species. Sequence homology is indicated by percent identity. CMT2E-linked head domain mutations NF- $\mathrm{L}^{\mathrm{P} 8 \mathrm{R}}$ and NF- $\mathrm{L}^{\mathrm{P} 22 \mathrm{~S}}$ are identified with an arrow with the corresponding amino acid substitution. These two mutations are located adjacent to phosphorylation sites. Notice that all but one $\left(\mathrm{ser}^{69}\right)$ bovine phosphorylation site is conserved between species. Sequence accession numbers used to generate this figure are as follows: Human NP_006149, Bovine NP_776546, Orangutan NP_001126494, Mouse NP_035040, and Rat NP_113971.

6.1. Amyotrophic Lateral Sclerosis (ALS). ALS is an adultonset NDD that selectively kills upper and lower motor neurons. Despite common pathogenic features, there is no identifiable genetic linkage in $90-95 \%$ of ALS cases (sporadic ALS) while $5-10 \%$ of ALS occurrences result from dominantly inherited genetic mutations (familial ALS) [63]. Dominant missense mutations in the gene for the cytoplasmic $\mathrm{Cu} / \mathrm{Zn}$ superoxide dismutase 1 (SOD1) are responsible for $20 \%$ of familial ALS cases [64]. Motor neuron cell bodies contain NF accumulations in cases of sporadic [39] and familial ALS [65].

Mice expressing SOD1-linked mutations resulted in motor neuron pathology that included ectopic accumulations of phosphorylated NFs within lumbar motor neuron cell bodies [63]. Furthermore, SOD1-linked ALS mouse models demonstrated impaired slow axonal transport of NFs as the earliest indication of pathology [66]. In order to identify the role of NFs in SOD1-linked ALS pathogenesis, $\mathrm{SOD} 1^{\mathrm{G} 85 \mathrm{R}} / \mathrm{NF}^{-\mathrm{L}^{-1-}}$ mice were generated [45]. The absence of assembled NFs in axons (NF- $\mathrm{L}^{-/-}$) alleviated the selective motor neuron vulnerability and slowed $\mathrm{SOD} 1{ }^{\mathrm{G} 85 \mathrm{R}}$-mediated toxicity. Furthermore, elimination of NF KSP motifs via single or double truncation of NF-M and NF-H C-termini delayed disease onset and extended survival of the SOD $1^{\mathrm{G} 37 \mathrm{R}}$ transgenic mouse model [67]. Interestingly, delayed onset and enhanced survival were additive with truncation of both NF-M and NF-H C-termini enhancing survival better than truncation of a single NF C-terminus [67]. Furthermore, preventing NF KSP phosphorylation through C-terminal truncation of NF-M and NF-H enhanced motor neuron survival in SOD1 ${ }^{\mathrm{G} 37 \mathrm{R}}$ mice [67]. These results suggested that the NF network, particularly NF-M and NF-H C-termini and their phosphorylation, contributes to ALS pathogenesis. However, the mechanism by which removal of axonal NFs or preventing their C-terminal phosphorylation ameliorated ALS pathology remains to be determined.

6.2. Charcot-Marie-Tooth (CMT). CMT is the most commonly inherited peripheral neuropathy. Based upon the specific genetic defect CMTs are grouped into four main types, CMT1-4 with each type having several subtypes $[68,69]$. CMT2E has been linked to mutations throughout the functional domains of NF-L [70] (Figure 3(A)). 
NF-L mutations are typically autosomal dominant though two recessive forms have been identified [71, 72] (Figure 3(A)). CMT2E recessive mutations introduce a nonsense mutation resulting in loss of NF-L protein.

Two CMT2E-linked NF-L mutations are located at positions, $\mathrm{Pro}^{8}$ and $\mathrm{Pro}^{22}$. These mutations are located adjacent to known bovine head domain phosphorylation sites, $\mathrm{Ser}^{2}$, $\mathrm{Ser}^{12}, \mathrm{Ser}^{26}$, and $\mathrm{Ser}^{27}$ (Figure 3(B)). Interestingly, $\mathrm{Pro}^{8}$ and $\mathrm{Pro}^{22}$ along with all $\mathrm{N}$-terminal serine residues are highly conserved across mammals [73] (Figure 3(B)). The bovine NF-L head domain contains thirteen phosphorylation sites [73]. All but one of these sites (Ser ${ }^{69}$ ) have complete sequence homology in NF-L from a variety of mammals [73] (Figure 3(B)).

When expressed in cultured cells, the hNF-L ${ }^{\text {P22S/T }}$ mutation abolished NF-L head domain phosphorylation [74]. Additionally, mutation of a proline residue in keratin 8 prevented phosphorylation of a nearby serine residue [60]. Taken together, these data suggest that NF- $\mathrm{L}^{\mathrm{P} 8 \mathrm{R}}$ and NF- $\mathrm{L}^{\mathrm{P} 22 \mathrm{~S}}$ mutations may prevent $\mathrm{N}$-terminal phosphorylation. As previously discussed, expressing NF-L that mimics constitutive phosphorylation of an N-terminal serine, NF-L ${ }^{\text {S55D }}$, prevented NF assembly [75], and N-terminal phosphorylation of NF-M prevented C-terminal phosphorylation [7]. Based on the previously described role of NF phosphorylation, abolished $\mathrm{N}$-terminal phosphorylation would result in ectopic NF assembly and phosphorylation [7, 8, 75], altered NF transport $[21,26,30,33,35]$, and ectopic NF accumulations $[7,8,75]$. Consistent with this prediction, transgenic mice expressing the CMT-linked head domain mutation, hNF-L ${ }^{\text {P22S }}$, displayed disrupted axonal transport [76]. Furthermore, mutations within a conserved sequence at the end of the rod domain, hNF-L $\mathrm{L}^{\mathrm{E} 397 \mathrm{~K}}$, also resulted in ectopic NF phosphorylation as early as 1 month [77].

6.3. Spinal Muscular Atrophy (SMA). SMA is an autosomal recessive disorder that is the leading genetic cause of infantile death [78]. SMA is caused by a deficiency in survival motor neuron (SMN) protein levels produced by the SMN1 gene $[79,80]$, which is a ubiquitously expressed protein that has a well-described role in RNA metabolism [81-85]. Deficiencies in SMN protein levels lead to skeletal muscle paralysis [86]. The severity of SMA is dependent on the relative copy number of the SMN2 gene which produces $\sim 15 \%$ functional SMN that compensates for the loss of SMN1 [87, 88]. As a result, SMA has a broad disease spectrum made up of SMA type 0, I, II, III, and IV [89].

Within motor neurons, SMA patients [90] developed phosphorylated NF accumulations within NMJs. Similar NF accumulations were one of the earliest pathological alterations observed in SMA mouse models [91-94]. The cause and pathogenic properties of NF accumulations are poorly understood in SMA. However, recent work demonstrated that NF accumulations were likely a result of local NF alterations [95]. Ectopic NF accumulations within motor neuron cell bodies were not apparent early or late in disease [91]. Moreover, the NF network was not altered in proximal segments of motor axons of the fifth lumbar ventral root
[95], which is one of three lumbar segments that together form the sciatic nerve. Interestingly, mice with alterations to the UPS displayed phosphorylated NF accumulations [49] strikingly similar to those observed in SMA models [9093]. Taken together, these results suggest that mechanisms disrupting NF turnover within motor neurons may be responsible for NF accumulations within NMJ of SMA patients. One potential NF alteration that could lead to reduced NF turnover and subsequent accumulation is altered NF phosphorylation.

\section{Conclusions}

NFs are abundant cytoskeletal proteins that undergo various PTMs. The most abundant PTM is phosphorylation of NF subunit proteins within myelinated axons. Phosphorylation of NFs was initially documented nearly 30 years ago and thought to regulate radial growth within myelinated axons. However, a series of recent analyses conducted on genetically modified mice has provided evidence against the role of NF phosphorylation in radial growth. Current evidence suggests that NF phosphorylation is both spatially and temporally regulated, which may be a mechanism to regulate NF assembly and accumulation. The presence of phosphorylated NFs in human NDDs suggests that altered NF phosphorylation dynamics may contribute to aberrant NF accumulation. Therefore, understanding the role of and mechanisms regulating NF PTM may prove critical to our understanding of the development and functioning of the nervous system in both healthy and diseased neurons.

\section{Conflict of Interests}

The authors declare that they have no conflict of interests.

\section{Acknowledgments}

This work was supported by grants from the National Institutes of Health Grant no. NS060073, Charcot-MarieTooth Association Grant no. C00014627, and University of Missouri Research Board to MLG. Salary support for M. L. Garcia was provided by the University of Missouri-Columbia and the C. S. Bond Life Sciences Center. J. M. Dale was supported by an ARRA supplement to T32 GM008396.

\section{References}

[1] R. L. Friede and T. Samorajski, "Axon caliber related to neurofilaments and microtubules in sciatic nerve fibers of rats and mice," Anatomical Record, vol. 167, no. 4, pp. 379-387, 1970.

[2] P. M. Steinert and D. R. Roop, "Molecular and cellular biology of intermediate filaments," Annual Review of Biochemistry, vol. 57, pp. 593-625, 1988.

[3] J. P. Julien and W. E. Mushynski, "A comparison of in vitroand in vivo-phosphorylated neurofilament polypeptides," Journal of Neurochemistry, vol. 37, no. 6, pp. 1579-1585, 1981.

[4] J. P. Julien and W. E. Mushynski, "Multiple phosphorylation sites in mammalian neurofilament polypeptides," Journal of Biological Chemistry, vol. 257, no. 17, pp. 10467-10470, 1982. 
[5] J. P. Julien and W. E. Mushynski, "The distribution of phosphorylation sites among identified proteolytic fragments of mammalian neurofilaments," Journal of Biological Chemistry, vol. 258, no. 6, pp. 4019-4025, 1983.

[6] Z. S. Xu, W. S. Liu, and M. B. Willard, "Identification of six phosphorylation sites in the $\mathrm{COOH}$-terminal tail region of the rat neurofilament protein M," Journal of Biological Chemistry, vol. 267, no. 7, pp. 4467-4471, 1992.

[7] Y. L. Zheng, B. S. Li, Veeranna, and H. C. Pant, "Phosphorylation of the head domain of neurofilament protein (NF-M). A factor regulating topographic phosphorylation of NF-M tail domain KSP sites in neurons," Journal of Biological Chemistry, vol. 278, no. 26, pp. 24026-24032, 2003.

[8] B. J. M. Gibb, J. P. Brion, J. Brownlees, B. H. Anderton, and C. C. J. Miller, "Neuropathological abnormalities in transgenic mice harbouring a phosphorylation mutant neurofilament transgene," Journal of Neurochemistry, vol. 70, no. 2, pp. 492 500,1998

[9] M. L. Garcia, M. V. Rao, J. Fujimoto et al., "Phosphorylation of highly conserved neurofilament medium KSP repeats is not required for myelin-dependent radial axonal growth," Journal of Neuroscience, vol. 29, no. 5, pp. 1277-1284, 2009.

[10] S. G. Waxman and M. V. Bennett, "Relative conduction velocities of small myelinated and non-myelinated fibres in the central nervous system," Nature, vol. 238, no. 85, pp. 217-219, 1972.

[11] M. H. Brill, S. G. Waxman, J. W. Moore, and R. W. Joyner, "Conduction velocity and spike configuration in myelinated fibres: computed dependence on internode distance," Journal of Neurology Neurosurgery and Psychiatry, vol. 40, no. 8, pp. 769-774, 1977.

[12] W. A. Rushton, "A theory of the effects of fibre size in medullated nerve," Journal of Physiology, vol. 115, no. 1, pp. 101-122, 1951.

[13] O. Ohara, Y. Gahara, T. Miyake, H. Teraoka, and T. Kitamura, "Neurofilament deficiency in quail caused by nonsense mutation in neurofilament-L gene," Journal of Cell Biology, vol. 121, no. 2, pp. 387-395, 1993.

[14] Q. Zhu, S. Couillard-Després, and J. P. Julien, "Delayed maturation of regenerating myelinated axons in mice lacking neurofilaments," Experimental Neurology, vol. 148, no. 1, pp. 299-316, 1997.

[15] S. M. De Waegh, V. M. Y. Lee, and S. T. Brady, "Local modulation of neurofilament phosphorylation, axonal caliber, and slow axonal transport by myelinating Schwann cells," Cell, vol. 68, no. 3, pp. 451-463, 1992.

[16] S. T. Hsieh, T. O. Crawford, and J. W. Griffin, "Neurofilament distribution and organization in the myelinated axons of the peripheral nervous system," Brain Research, vol. 642, no. 1-2, pp. 316-326, 1994.

[17] X. Yin, T. O. Crawford, J. W. Griffin et al., "Myelin-associated glycoprotein is a myelin signal that modulates the caliber of myelinated axons," Journal of Neuroscience, vol. 18, no. 6, pp. 1953-1962, 1998.

[18] G. A. Elder, V. L. Friedrich, P. Bosco et al., "Absence of the midsized neurofilament subunit decreases axonal calibers, levels of light neurofilament (NF-L), and neurofilament content," Journal of Cell Biology, vol. 141, no. 3, pp. 727-739, 1998.

[19] M. V. Rao, M. K. Houseweart, T. L. Williamson, T. O. Crawford, J. Folmer, and D. W. Cleveland, "Neurofilamentdependent radial growth of motor axons and axonal organization of neurofilaments does not require the neurofilament heavy subunit (NF-H) or its phosphorylation," Journal of Cell Biology, vol. 143, no. 1, pp. 171-181, 1998.
[20] G. A. Elder, V. L. Friedrich, C. Kang et al., "Requirement of heavy neurofilament subunit in the development of axons with large calibers," Journal of Cell Biology, vol. 143, no. 1, pp. 195205, 1998.

[21] Q. Zhu, M. Lindenbaum, F. Levavasseur, H. Jacomy, and J. P. Julien, "Disruption of the NF-H gene increases axonal microtubule content and velocity of neurofilament transport: relief of axonopathy resulting from the toxin $\beta, \beta^{\prime}$ iminodipropionitrile," Journal of Cell Biology, vol. 143, no. 1, pp. 183-193, 1998.

[22] M. V. Rao, M. L. Garcia, Y. Miyazaki et al., "Gene replacement in mice reveals that the heavily phosphorylated tail of neurofilament heavy subunit does not affect axonal caliber or the transit of cargoes in slow axonal transport," Journal of Cell Biology, vol. 158, no. 4, pp. 681-693, 2002.

[23] M. L. Garcia, C. S. Lobsiger, S. B. Shah et al., "NF-M is an essential target for the myelin-directed "outside-in" signaling cascade that mediates radial axonal growth," Journal of Cell Biology, vol. 163, no. 5, pp. 1011-1020, 2003.

[24] R. A. Nixon and S. E. Lewis, "Phosphorylation and dephosphorylation of neurofilament proteins in retinal ganglion cell neurons in vivo," Advances in Experimental Medicine and Biology, vol. 221, pp. 167-186, 1987.

[25] R. A. Nixon, P. A. Paskevich, R. K. Sihag, and C. Y. Thayer, "Phosphorylation on carboxyl terminus domains of neurofilament proteins in retinal ganglion cell neurons in vivo: influences on regional neurofilament accumulation, interneurofilament spacing, and axon caliber," Journal of Cell Biology, vol. 126, no. 4, pp. 1031-1046, 1994.

[26] J. Motil, W. K. H. Chan, M. Dubey et al., "Dynein mediates retrograde neurofilament transport within axons and anterograde delivery of NFs from perikarya into axons: regulation by multiple phosphorylation events," Cell Motility and the Cytoskeleton, vol. 63, no. 5, pp. 266-286, 2006.

[27] H. C. Pant and Veeranna, "Neurofilament phosphorylation," Biochemistry and Cell Biology, vol. 73, no. 9-10, pp. 575-592, 1995.

[28] I. Sánchez, L. Hassinger, R. K. Sihag, D. W. Cleveland, P. Mohan, and R. A. Nixon, "Local control of neurofilament accumulation during radial growth of myelinating axons in vivo: selective role of site-specific phosphorylation," Journal of Cell Biology, vol. 151, no. 5, pp. 1013-1024, 2000.

[29] J. T. Yabe, C. Jung, W. K. H. Chan, and T. B. Shea, "Phosphodependent association of neurofilament proteins with kinesin in situ," Cell Motility and the Cytoskeleton, vol. 45, no. 4, pp. 249-262, 2000.

[30] C. Jung, S. Lee, D. Ortiz, Q. Zhu, J. P. Julien, and T. B. Shea, "The high and middle molecular weight neurofilament subunits regulate the association of neurofilaments with kinesin: inhibition by phosphorylation of the high molecular weight subunit," Molecular Brain Research, vol. 141, no. 2, pp. 151-155, 2005.

[31] J. T. Yabe, A. Pimenta, and T. B. Shea, "Kinesin-mediated transport of neurofilament protein oligomers in growing axons," Journal of Cell Science, vol. 112, no. 21, pp. 3799-3814, 1999.

[32] H. Jacomy, Q. Zhu, S. Couillard-Després, J. M. Beaulieu, and J. P. Julien, "Disruption of type IV intermediate filament network in mice lacking the neurofilament medium and heavy subunits," Journal of Neurochemistry, vol. 73, no. 3, pp. 972984, 1999.

[33] M. V. Rao, J. Campbell, A. Yuan et al., "The neurofilament middle molecular mass subunit carboxyl-terminal tail domains is essential for the radial growth and cytoskeletal 
architecture of axons but not for regulating neurofilament transport rate," Journal of Cell Biology, vol. 163, no. 5, pp. 1021-1031, 2003.

[34] A. Yuan, R. A. Nixon, and M. V. Rao, "Deleting the phosphorylated tail domain of the neurofilament heavy subunit does not alter neurofilament transport rate in vivo," Neuroscience Letters, vol. 393, no. 2-3, pp. 264-268, 2006.

[35] D. M. Yates, C. Manser, K. J. De Vos, C. E. Shaw, D. M. McLoughlin, and C. C. J. Miller, "Neurofilament subunit (NFL) head domain phosphorylation regulates axonal transport of neurofilaments," European Journal of Cell Biology, vol. 88, no. 4, pp. 193-202, 2009.

[36] P. H. Tu, G. Elder, R. A. Lazzarini, D. Nelson, J. Q. Trojanowski, and V. M. Y. Lee, "Overexpression of the human NFM subunit in transgenic mice modifies the level of endogenous NFL and the phosphorylation state of NFH subunits," Journal of Cell Biology, vol. 129, no. 6, pp. 1629-1640, 1995.

[37] Z. Xu, L. C. Cork, J. W. Griffin, and D. W. Cleveland, "Increased expression of neurofilament subunit NF-L produces morphological alterations that resemble the pathology of human motor neuron disease," Cell, vol. 73, no. 1, pp. 2333, 1993.

[38] F. Cote, J. F. Collard, and J. P. Julien, "Progressive neuronopathy in transgenic mice expressing the human neurofilament heavy gene: a mouse model of amyotrophic lateral sclerosis," Cell, vol. 73, no. 1, pp. 35-46, 1993.

[39] A. Hirano, H. Donnenfeld, S. Sasaki, and I. Nakano, "Fine structural observations of neurofilamentous changes in amyotrophic lateral sclerosis," Journal of Neuropathology and Experimental Neurology, vol. 43, no. 5, pp. 461-470, 1984.

[40] A. Hirano, I. Nakano, and L. T. Kurland, "Fine structural study of neurofibrillary changes in a family with amyotrophic lateral sclerosis," Journal of Neuropathology and Experimental Neurology, vol. 43, no. 5, pp. 471-480, 1984.

[41] J. Meier, S. Couillard-Després, H. Jacomy, C. Gravel, and J. P. Julien, "Extra neurofilament NF-L subunits rescue motor neuron disease caused by overexpression of the human NF$\mathrm{H}$ gene in mice," Journal of Neuropathology and Experimental Neurology, vol. 58, no. 10, pp. 1099-1110, 1999.

[42] D. R. McLachlan, W. J. Lukiw, L. Wong, C. Bergeron, and N. T. Bech-Hansen, "Selective messenger RNA reduction in Alzheimer's disease," Brain Research, vol. 427, no. 3, pp. 255261, 1988.

[43] N. K. Y. Wong, B. P. He, and M. J. Strong, "Characterization of neuronal intermediate filament protein expression in cervical spinal motor neurons in sporadic amyotrophic lateral sclerosis (ALS)," Journal of Neuropathology and Experimental Neurology, vol. 59, no. 11, pp. 972-982, 2000.

[44] F. M. Menzies, A. J. Grierson, M. R. Cookson et al., "Selective loss of neurofilament expression in $\mathrm{Cu} / \mathrm{Zn}$ superoxide dismutase (SOD1) linked amyotrophic lateral sclerosis," Journal of Neurochemistry, vol. 82, no. 5, pp. 1118-1128, 2002.

[45] T. L. Williamson, L. I. Bruijn, Q. Zhu et al., "Absence of neurofilaments reduces the selective vulnerability of motor neurons and slows disease caused by a familial amyotrophic lateral sclerosis-linked superoxide dismutase 1 mutant," Proceedings of the National Academy of Sciences of the United States of America, vol. 95, no. 16, pp. 9631-9636, 1998.

[46] M. Balastik, F. Ferraguti, A. Pires-da Silva et al., "Deficiency in ubiquitin ligase TRIM2 causes accumulation of neurofilament light chain and neurodegeneration," Proceedings of the National Academy of Sciences of the United States of America, vol. 105, no. 33, pp. 12016-12021, 2008.
[47] Q. Wang, F. Song, C. Zhang et al., "Carboxyl-terminus of Hsc70 interacting protein mediates 2,5-hexanedione-induced neurofilament medium chain degradation," Biochemical Pharmacology, vol. 81, no. 6, pp. 793-799, 2011.

[48] J. P. Gou and J. F. Leterrier, "Possible involvement of ubiquitination in neurofilament degradation," Biochemical and Biophysical Research Communications, vol. 217, no. 2, pp. 529$538,1995$.

[49] P. C. Chen, L. N. Qin, X. M. Li et al., "The proteasomeassociated deubiquitinating enzyme Usp14 is essential for the maintenance of synaptic ubiquitin levels and the development of neuromuscular junctions," Journal of Neuroscience, vol. 29, no. 35, pp. 10909-10919, 2009.

[50] S. M. Wilson, B. Bhattacharyya, R. A. Rachel et al., "Synaptic defects in ataxia mice result from a mutation in Usp14, encoding a ubiquitin-specific protease," Nature Genetics, vol. 32, no. 3, pp. 420-425, 2002.

[51] C. J. D'Amato and S. P. Hicks, "Neuropathologic alterations in the ataxia (paralytic) mouse," Archives of Pathology, vol. 80, no. 6, pp. 604-612, 1965.

[52] S. Crimmins, Y. Jin, C. Wheeler et al., "Transgenic rescue of ataxia mice with neuronal-specific expression of ubiquitinspecific protease 14," Journal of Neuroscience, vol. 26, no. 44, pp. 11423-11431, 2006.

[53] R. Verma and R. J. Deshaies, "A proteasome howdunit: the case of the missing signal," Cell, vol. 101, no. 4, pp. 341-344, 2000.

[54] S. Thompson, A. N. Pearson, M. D. Ashley et al., "Identification of a novel Bcl-2-interacting mediator of cell death (Bim) E3 ligase, tripartite motif-containing protein 2 (TRIM2), and its role in rapid ischemic tolerance-induced neuroprotection," Journal of Biological Chemistry, vol. 286, no. 22, pp. 1933119339, 2011.

[55] I. Rees, S. Lee, H. Kim, and F. T. F. Tsai, “The E3 ubiquitin ligase CHIP binds the androgen receptor in a phosphorylationdependent manner," Biochimica et Biophysica Acta, vol. 1764, no. 6, pp. 1073-1079, 2006.

[56] Q. Sun, R. A. Jackson, C. Ng, G. R. Guy, and J. Sivaraman, "Additional serine/threonine phosphorylation reduces binding affinity but preserves interface topography of substrate proteins to the c-Cbl TKB domain," PLoS ONE, vol. 5, no. 9, Article ID e12819, pp. 1-11, 2010.

[57] T. B. Shea, P. A. Paskevich, and M. L. Beermann, "The protein phosphatase inhibitor okadaic acid increases axonal neurofilaments and neurite caliber, and decreases axonal microtubules in NB2a/d1 cells," Journal of Neuroscience Research, vol. 35, no. 5, pp. 507-521, 1993.

[58] C. X. Gong, J. Z. Wang, K. Iqbal, and I. Grundke-Iqbal, "Inhibition of protein phosphatase $2 \mathrm{~A}$ induces phosphorylation and accumulation of neurofilaments in metabolically active rat brain slices," Neuroscience Letters, vol. 340, no. 2, pp. 107110, 2003.

[59] H. C. Pant, "Dephosphorylation of neurofilament proteins enhances their susceptibility to degradation by calpain," Biochemical Journal, vol. 256, no. 2, pp. 665-668, 1988.

[60] N. O. Ku and M. B. Omary, "Keratins turn over by ubiquitination in a phosphorylation-modulated fashion," Journal of Cell Biology, vol. 149, no. 3, pp. 547-552, 2000.

[61] N. Lüdemann, A. Clement, V. H. Hans, J. Leschik, C. Behl, and R. Brandt, "O-glycosylation of the tail domain of neurofilament protein $\mathrm{M}$ in human neurons and in spinal cord tissue of a rat model of amyotrophic lateral sclerosis (ALS)," Journal of Biological Chemistry, vol. 280, no. 36, pp. 3164831658, 2005. 
[62] Y. Deng, B. Li, F. Liu et al., "Regulation between OGlcNAcylation and phosphorylation of neurofilament-M and their dysregulation in Alzheimer disease," FASEB Journal, vol. 22, no. 1, pp. 138-145, 2008.

[63] D. W. Cleveland and J. D. Rothstein, "From Charcot to Lou Gehrig: deciphering selective motor neuron death in ALS," Nature Reviews Neuroscience, vol. 2, no. 11, pp. 806-819, 2001.

[64] D. R. Rosen, T. Siddique, D. Patterson et al., "Mutations in $\mathrm{Cu} / \mathrm{Zn}$ superoxide dismutase gene are associated with familial amyotrophic lateral sclerosis," Nature, vol. 362, no. 6415, pp. 59-62, 1993.

[65] G. A. Rouleau, A. W. Clark, K. Rooke et al., "SOD1 mutation is associated with accumulation of neurofilaments in amyotrophic lateral sclerosis," Annals of Neurology, vol. 39, no. 1, pp. 128-131, 1996.

[66] B. Zhang, P. H. Tu, F. Abtahian, J. Q. Trojanowski, and V. M. Y. Lee, "Neurofilaments and orthograde transport are reduced in ventral root axons of transgenic mice that express human SOD1 with a G93A mutation," Journal of Cell Biology, vol. 139, no. 5, pp. 1307-1315, 1997.

[67] C. S. Lobsiger, M. L. Garcia, C. M. Ward, and D. W. Cleveland, "Altered axonal architecture by removal of the heavily phosphorylated neurofilament tail domains strongly slows superoxide dismutase 1 mutant-mediated ALS," Proceedings of the National Academy of Sciences of the United States of America, vol. 102, no. 29, pp. 10351-10356, 2005.

[68] H. Skre, "Genetic and clinical aspects of Charcot Marie Tooth's disease," Clinical Genetics, vol. 6, no. 2, pp. 98-118, 1974.

[69] A. E. H. Emery, "Population frequencies of inherited neuromuscular diseases—a world survey," Neuromuscular Disorders, vol. 1, no. 1, pp. 19-29, 1991.

[70] D. M. Barry, S. Millecamps, J. P. Julien, and M. L. Garcia, "New movements in neurofilament transport, turnover and disease," Experimental Cell Research, vol. 313, no. 10, pp. 2110-2120, 2007.

[71] S. W. Yum, J. Zhang, K. Mo, J. Li, and S. S. Scherer, "A novel recessive Nefl mutation causes a severe, early-onset axonal neuropathy," Annals of neurology, vol. 66, no. 6, pp. 759-770, 2009.

[72] A. Abe, C. Numakura, K. Saito et al., "Neurofilament light chain polypeptide gene mutations in Charcot-Marie-Tooth disease: nonsense mutation probably causes a recessive phenotype," Journal of Human Genetics, vol. 54, no. 2, pp. 94-97, 2009.

[73] R. Hashimoto, Y. Nakamura, S. Komai et al., "Site-specific phosphorylation of neurofilament- $\mathrm{L}$ is mediated by calcium/calmodulin-dependent protein kinase II in the apical dendrites during long-term potentiation," Journal of Neurochemistry, vol. 75, no. 1, pp. 373-382, 2000.

[74] T. Sasaki, T. Gotow, M. Shiozaki et al., "Aggregate formation and phosphorylation of neurofilament-L Pro22 CharcotMarie-Tooth disease mutants," Human Molecular Genetics, vol. 15, no. 6, pp. 943-952, 2006.

[75] B. J. M. Gibb, J. Robertson, and C. C. J. Miller, "Assembly properties of neurofilament light chain Ser55 mutants in transfected mammalian cells," Journal of Neurochemistry, vol. 66, no. 3, pp. 1306-1311, 1996.

[76] F. Dequen, M. Filali, R. C. Larivière, R. Perrot, S. I. Hisanaga, and J. P. Julien, "Reversal of neuropathy phenotypes in conditional mouse model of Charcot-Marie-Tooth disease type 2E," Human Molecular Genetics, vol. 19, no. 13, Article ID ddq149, pp. 2616-2629, 2010.

[77] H. Shen, D. M. Barry, J. M. Dale, V. B. Garcia, N. A. Calcutt, and M. L. Garcia, "Muscle pathology without severe nerve pathology in a new mouse model of charcot-marie-tooth disease type 2E," Human Molecular Genetics, vol. 20, no. 13, pp. 2535-2548, 2011.

[78] J. Pearn, "Incidence, prevalence, and gene frequency studies of chronic childhood spinal muscular atrophy," Journal of Medical Genetics, vol. 15, no. 6, pp. 409-413, 1978.

[79] U. R. Monani, D. D. Coovert, and A. H. M. Burghes, "Animal Models of spinal muscular atrophy," Human Molecular Genetics, vol. 9, no. 16, pp. 2451-2457, 2000.

[80] U. R. Monani, M. Sendtner, D. D. Coovert et al., "The human centromeric survival motor neuron gene (SMN2) rescues embryonic lethality in $\operatorname{Smn}(-/-)$ mice and results in a mouse with spinal muscular atrophy," Human Molecular Genetics, vol. 9, no. 3, pp. 333-339, 2000.

[81] L. Pellizzoni, N. Kataoka, B. Charroux, and G. Dreyfuss, "A novel function for SMN, the spinal muscular atrophy disease gene product, in pre-mRNA splicing," Cell, vol. 95, no. 5, pp. 615-624, 1998.

[82] D. Bühler, V. Raker, R. Lührmann, and U. Fischer, "Essential role for the tudor domain of SMN in spliceosomal U snRNP assembly: implications for spinal muscular atrophy," Human Molecular Genetics, vol. 8, no. 13, pp. 2351-2357, 1999.

[83] G. Meister, D. Bühler, R. Pillai, F. Lottspeich, and U. Fischer, "A multiprotein complex mediates the ATP-dependent assembly of spliceosomal U snRNPs," Nature Cell Biology, vol. 3, no. 11, pp. 945-949, 2001.

[84] F. Gabanella, M. E. R. Butchbach, L. Saieva, C. Carissimi, A. H. M. Burghes, and L. Pellizzoni, "Ribonucleoprotein assembly defects correlate with spinal muscular atrophy severity and preferentially affect a subset of spliceosomal snRNPs," PLoS ONE, vol. 2, no. 9, article e921, 2007.

[85] Z. Zhang, F. Lotti, K. Dittmar et al., "SMN deficiency causes tissue-specific perturbations in the repertoire of snRNAs and widespread defects in splicing," Cell, vol. 133, no. 4, pp. 585600, 2008.

[86] J. H. Pearn, D. Gardner-Medwin, and J. Wilson, "A clinical study of chronic childhood spinal muscular atrophy. A review of 141 cases," Journal of the Neurological Sciences, vol. 38, no. 1, pp. 23-37, 1978.

[87] M. Feldkötter, V. Schwarzer, R. Wirth, T. F. Wienker, and B. Wirth, "Quantitative analyses of SMN1 and SMN2 based on real-time lightcycler PCR: fast and highly reliable carrier testing and prediction of severity of spinal muscular atrophy," American Journal of Human Genetics, vol. 70, no. 2, pp. 358$368,2002$.

[88] S. Lefebvre, P. Burlet, Q. Liu et al., "Correlation between severity and SMN protein level in spinal muscular atrophy," Nature Genetics, vol. 16, no. 3, pp. 265-269, 1997.

[89] B. S. Russman, "Spinal muscular atrophy: clinical classification and disease heterogeneity," Journal of Child Neurology, vol. 22, no. 8, pp. 946-951, 2007.

[90] C. Cifuentes-Diaz, S. Nicole, M.E. Velasco et al., "Neurofilament accumulation at the motor endplate and lack of axonal sprouting in a spinal muscular atrophy mouse model," Human Molecular Genetics, vol. 11, no. 12, pp. 1439-1447, 2002.

[91] S. Kariya, G. H. Park, Y. Maeno-Hikichi et al., "Reduced SMN protein impairs maturation of the neuromuscular junctions in mouse models of spinal muscular atrophy," Human Molecular Genetics, vol. 17, no. 16, pp. 2552-2569, 2008.

[92] L. M. Murray, L. H. Comley, D. Thomson, N. Parkinson, K. Talbot, and T. H. Gillingwater, "Selective vulnerability of motor neurons and dissociation of pre- and post-synaptic pathology at the neuromuscular junction in mouse models of 
spinal muscular atrophy," Human Molecular Genetics, vol. 17, no. 7, pp. 949-962, 2008.

[93] L. Kong, X. Wang, D. W. Choe et al., "Impaired synaptic vesicle release and immaturity of neuromuscular junctions in spinal muscular atrophy mice," Journal of Neuroscience, vol. 29, no. 3, pp. 842-851, 2009.

[94] E. Dachs, M. Hereu, L. Piedrafita, A. Casanovas, J. Calderó, and J. E. Esquerda, "Defective neuromuscular junction organization and postnatal myogenesis in mice with severe spinal muscular atrophy," Journal of Neuropathology and Experimental Neurology, vol. 70, no. 6, pp. 444-461, 2011.

[95] J. M. Dale, H. Shen, D. M. Barry et al., "The spinal muscular atrophy mouse model, $\operatorname{SMA} \Delta 7$, displays altered axonal transport without global neurofilament alterations," Acta Neuropathologica, vol. 122, no. 3, pp. 331-341, 2011. 

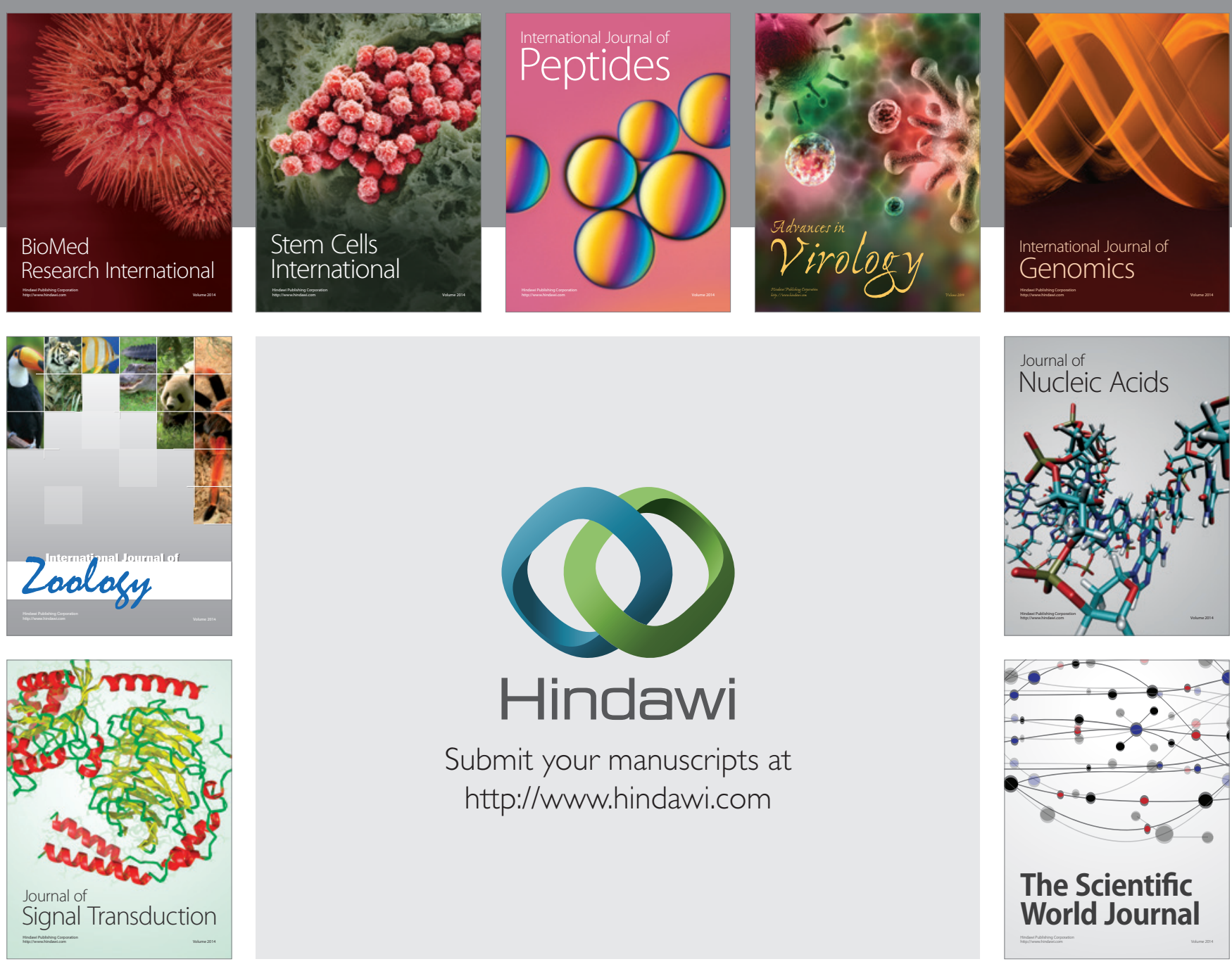

Submit your manuscripts at

http://www.hindawi.com
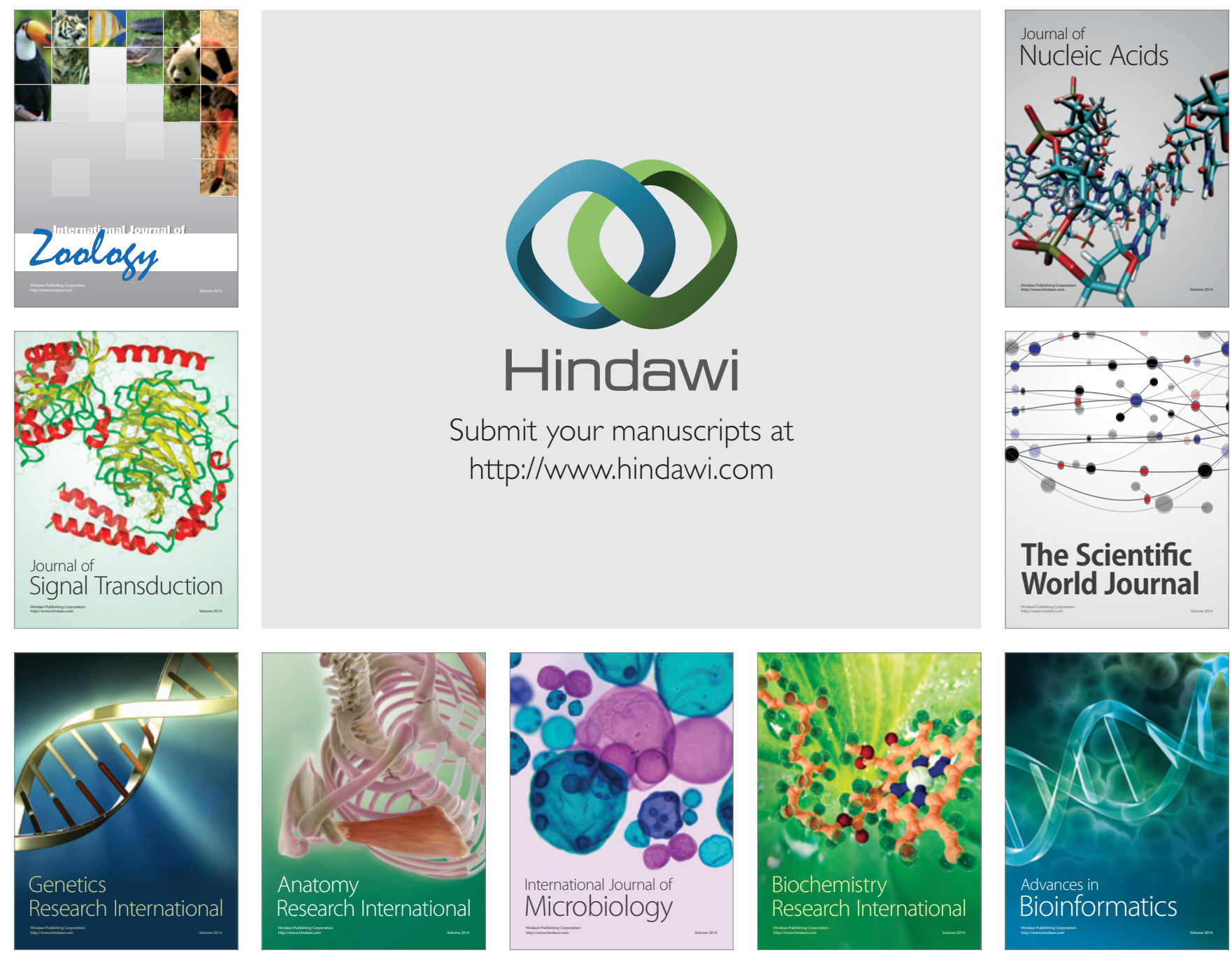

The Scientific World Journal
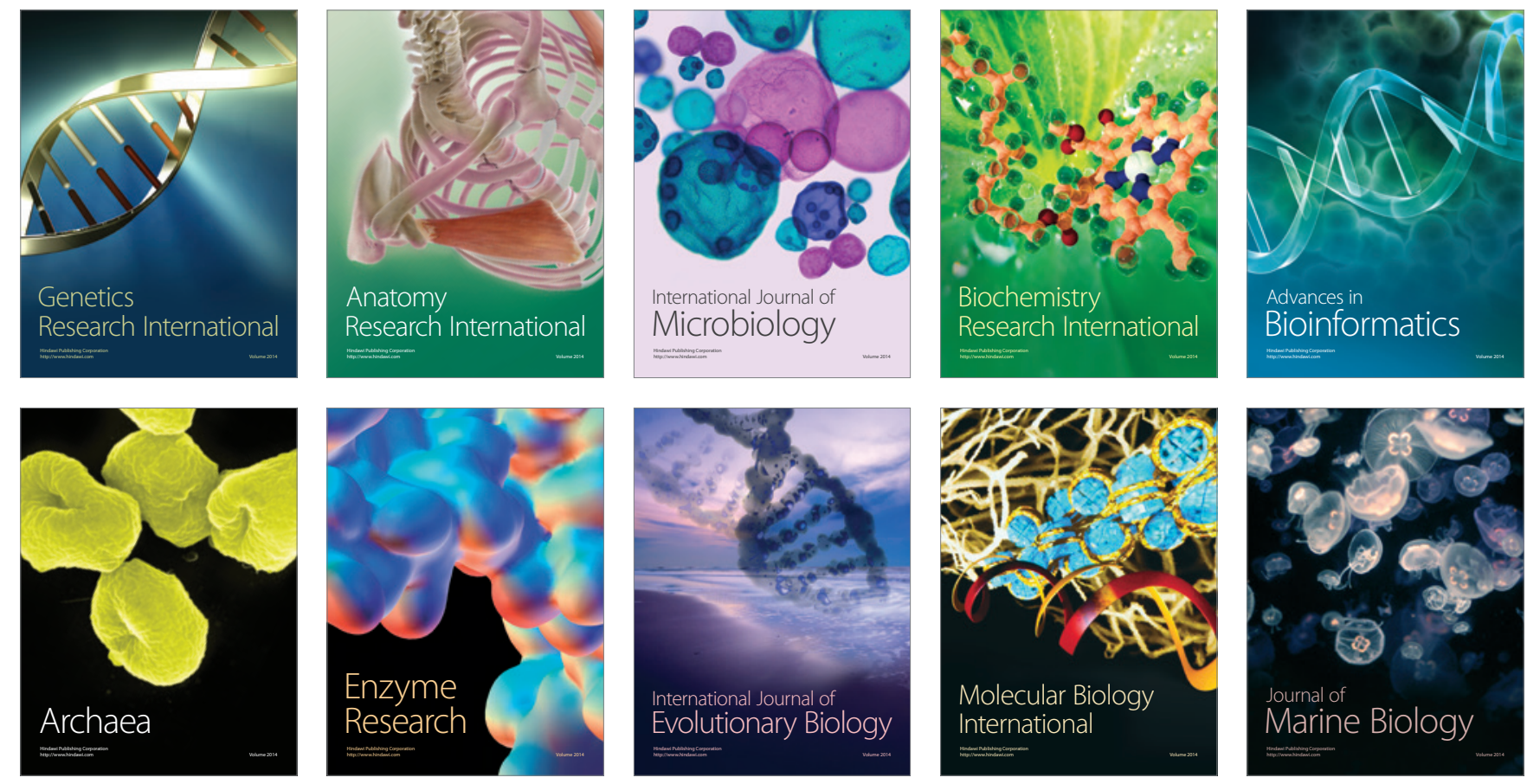